Motivational modulation of rhythms of the expression of the clock protein, PER2, in the limbic forebrain

Shimon Amir and Jane Stewart

Center for Studies in Behavioral Neurobiology/Groupe de Recherche en

Neurobiologie Comportementale

Department of Psychology, Concordia University

Montreal, QC, Canada

Corresponding author:

Shimon Amir

Center for Studies in Behavioral Neurobiology

Department of Psychology, Concordia University

7141 Sherbrooke St. W

Montreal, QC, H4B 1R6

Email: Shimon.amir@concordia.ca

Word count

Abstract: 154

Text: 3285

References: 71

Figures: 1

Suppl material: 0 


\begin{abstract}
Key molecular components of the mammalian circadian clock are expressed rhythmically in many brain areas and peripheral tissues in mammals. Here we review findings from our work on rhythms of expression of the clock protein, Period2 (PER2), in four regions of the limbic forebrain known to be important in the regulation of motivational and emotional states. These regions include the oval nucleus of the bed nucleus of the stria terminalis (BNSTov), the central nucleus of the amygdala (CEA), the basolateral amygdala (BLA) and the dentate gyrus (DG). Daily rhythms in the expression of PER2 in these regions are controlled by the master circadian pacemaker, the suprachiasmatic nucleus (SCN) but, importantly, they are also sensitive to homeostatic perturbations and to hormonal states that directly influence motivated behavior. Thus, circadian information from the SCN and homeostatic signals are integrated in these regions of the limbic forebrain to affect the temporal organization of motivational and emotional process.
\end{abstract}

Key Words: Period2, oval nucleus of the bed nucleus of the stria terminalis, central nucleus of the amygdala, basolateral amygdala, dentate gyrus, suprachiasmatic nucleus, glucocorticoid, gonadal hormones, restricted feeding 
An intriguing but poorly appreciated finding is that circadian rhythms are modulated by motivational or emotional state. This involvement is demonstrated by experiments in animals (1) and humans (2) showing that stress disrupts circadian rhythmicity and by clinical evidence of altered physiological rhythms in affective disorders (3).

In our studies of the interface between motivational or emotional state and circadian rhythms, we showed, for example, that induction of fear in rats can disrupt photic resetting of the circadian clock, the suprachiasmatic nucleus of the hypothalamus (SCN). Specifically, we found that light-induced expression of the cellular activity marker, Fos, in the retinorecipient region of the SCN and lightinduced phase shifts in free-running activity rhythms are attenuated in rats exposed to light in a context that induced conditioned fear (4). Such conditioned fear also attenuates light-induced suppression of melatonin release (5), supporting the view that limbic forebrain mechanisms involved in emotional regulation interface with mechanisms that mediate the transmission of light to the $\mathrm{SCN}$. Consistent with this idea, we also found that when light, itself, is made a conditioned aversive stimulus, its ability to induce Fos expression in the SCN is reduced (6). The same conditioned aversive stimulus enhanced Fos expression in the primary visual pathways (7), pointing to the differential effect of aversive emotional events on neural processes underlying visual perception and those involved in circadian regulation. 
In more recent work we have found that many limbic forebrain nuclei implicated in the regulation of motivational or emotional state exhibit daily rhythms in expression of the circadian clock protein, Period2 (PER2) $(8,9)$. This finding, together with evidence that the rhythms in PER2 expression in two regions, the oval nucleus of bed nucleus of the stria terminalis (BNSTov) and central nucleus of the amygdala (CEA) are regulated by corticosterone, led us to propose that motivational events modulate circadian rhythms, not only indirectly via light input mechanisms upstream from the SCN, but also by directly modulating tissue specific circadian mechanisms located downstream from the SCN. In this paper we describe the nature of PER2 rhythms in the limbic forebrain and findings concerning the neural, hormonal and behavioral mechanisms that regulate and modulate these rhythms.

\section{Circadian clocks}

Current models of circadian organization in mammals posit a hierarchical system in which a light-entrainable master clock located in the SCN synchronizes networks of subordinate circadian oscillators in the brain and periphery. These subordinate oscillators are presumed to control, in a tissue-specific manner, the daily fluctuations in cellular and metabolic activity and functional output (10). At the cellular level, circadian rhythmicity is based on interlocked negative and positive transcription and translation/post-translation feedback loops that involve the products of at least 10 clock genes. These include two genes encoding helixloop-helix PAS transcription factors (Clock and Bmal1), three Period genes 
(Per1, Per2 and Per3), two Cryptochrome genes (Cry1 and Cry2), two orphan nuclear receptor genes (Rev-erb $\square$, Rora), and a gene encoding casein kinase (Tau) (11). Circadian rhythms in expression of clock genes and proteins have been described in the SCN as well as in many brain regions outside the SCN and in most peripheral tissues (12-14). Importantly, in spite of the resemblance in molecular composition, the rhythms of the SCN differ from those in other brain and peripheral tissues. In the SCN the rhythms are intrinsically synchronous and self-sustaining, whereas circadian rhythms in most other tissues dampen in the absence of synchronizing input $(12,14)$.

The mechanism whereby the SCN maintains and coordinates circadian oscillations in the rest of the brain and periphery is complex. Neural projections and diffusible peptides from the $\mathrm{SCN}$, as well as neural (such as the sympathetic nervous systems), endocrine (such as corticosterone, melatonin, epinephrine) and behavioral (such as locomotor activity, feeding) processes under SCN control have all been proposed as possible mediators $(15,16)$. Furthermore, multiple intracellular signaling molecules that can reset molecular clocks in the brain and periphery have been identified. These include glucose, cAMP, PKA, PKC, and MAPK $(17,18)$. Finally, circadian oscillations in clock gene expression in the brain and periphery can be modulated directly, downstream from the SCN. Thus, exposure to stress or drugs of abuse $(19,20)$, scheduled restricted feeding (21-24), exercise (25), and periodic absence of nursing mothers (26) have all been shown to either induce or shift the phase of clock gene expression in a number of brain structures and peripheral tissues in rodents. 


\section{PER2 rhythms reveal a novel circadian oscillators in the limbic forebrain}

The rhythmic expression of PER2, the protein product of the circadian clock gene, Per2, is a defining feature of clock cells in mammals (27). In mapping studies of the expression of PER2 in rat brain, we found daily rhythms in four anatomically and functionally related regions of the limbic forebrain: the oval nucleus of the bed nucleus of the stria terminalis (BNSTov, located in the dorsal lateral BNST), central nucleus of the amygdala (CEA), basolateral amygdala (BLA) and dentate gyrus (DG) $(8,9)$. This finding was of immediate interest because each of these regions is known to participate in the regulation of motivational and emotional state and to modulate specific behavioral and physiological processes. These include behavioral, endocrine and neural responses to stress and to drugs of abuse, regulation of feeding, anxiety, learning and memory, and reproductive and maternal behaviors (28-31). Significantly, we found that the PER2 rhythms in these regions follow two opposite temporal patterns. In the BNSTov and CEA, the expression of PER2 is maximal around the time of transition from day to night, and is in perfect synchrony with the PER2 rhythm of the SCN. In contrast, the rhythms of PER2 in BLA and DG peak in the morning in antiphase with the rhythms in the $S C N$, BNSTov and CEA (Fig. 1).

\section{PER2 rhythms in the limbic forebrain are normally synchronized by the SCN}


To understand the mechanisms that control the rhythms of PER2 in the limbic forebrain, we first assessed the role of the SCN. We found that bilateral SCN lesions, or prolonged housing in constant light (LL), which eliminate PER2 rhythms in the SCN and disrupt circadian behavioral rhythms, abolished the rhythm of PER2 in the BNSTov, CEA, BLA and DG $(8,9)$. These experiments confirmed the subordinate nature of these rhythms. In another experiment we found that unilateral SCN lesions, which do not affect circadian behavioral rhythms, blunted the rhythm of PER2 in the limbic forebrain ipsilateral, but not contralateral to the lesioned side, emphasizing the importance of neural connections between the SCN and PER2 oscillations in the limbic forebrain (8).

\section{PER2 rhythms in the BNSTOV and CEA depend on a rhythm of glucocorticoids}

In spite of the importance of the SCN, we found in a series of experiments that the rhythms of PER2 in the BNSTov and CEA, but not SCN, BLA or DG, are sensitive to changes in circulating hormones. We first examined the involvement of glucocorticoid hormone, corticosterone. The release of corticosterone from the adrenal glands follows a circadian rhythm that is under the control of the SCN (32) and glucocorticoid hormones have been shown to induce per gene expression in mouse liver, in vivo, and in cultured rat-1 fibroblasts (33). Cells in the BNSTov, CEA, BLA and DG express glucocorticoid receptors and exhibit a wide range of responses to endogenous and exogenous glucocorticoids (34-36). We found that surgical removal of the adrenal glands flattened the rhythm of 
expression of PER2 in the BNSTov and CEA without affecting PER2 rhythmicity in BLA and DG, indicating that glucocorticoids play a selective role in the regulation of PER2 expression in the BNSTov and CEA $(8,9)$. Subsequently, we found that in the absence of the adrenals, corticosterone replacement via the drinking water, which restores daily fluctuations in corticosterone levels, restores the rhythm of PER2 in the BNSTov and CEA (37). Corticosterone replacement via subcutaneous constant-release pellets has no effect, demonstrating the importance of circadian glucocorticoid signaling in PER2 rhythms in the BNSTov and CEA. Finally, we found that in a conditional mutant mouse devoid of glucocorticoid receptors in the brain (38), PER2 rhythms in the BNSTov and CEA are absent, consistent with the idea that the effect of circulating corticosterone on PER2 rhythms in these regions is mediated by central glucocorticoid receptors (39).

\section{Role of thyroid hormones}

Thyroid hormones have been implicated in the regulation of behavioral and physiological circadian rhythms based on the evidence that surgical removal of the thyroid and parathyroid glands or chemical induction of hypothyroidism alters circadian locomotor activity rhythms and blunts the daily fluctuations in circulating corticosterone and prolactin levels in rodents $(40,41)$. Furthermore thyroid hormones modulate the expression of clock genes in peripheral tissue and recent evidence suggests a role for thyroid hormone-sensitive orphan nuclear receptors, 
REV-ERBalpha and RORa in the transcriptional regulation of clock gene expression (42).

We found that surgical removal of the thyroid and parathyroid glands (TPX) disrupts PER2 rhythms, again, selectively in the BNSTov and CEA (43). Thyroid hormones such as thyroxine (T4) and triiodothyronine (T3) might affect PER2 expression by modulating the transcription of the Per2 gene, indirectly, by modulating the transcriptional activity of REV-ERBalpha and RORa. Indeed, both have been implicated in the transcriptional regulation of BMAL1, an essential and direct positive regulator of Per2 transcription in mammalian cells (42). However, because this mechanism is likely to be ubiquitous, affecting clock gene expression in all tissues, it cannot account for the selective effect of TPX on PER2 expression in the BNSTov and CEA. Other possible mechanisms are their effects on the daily rhythm of plasma corticosterone levels (41), or their effect on neurotransmitters and peptides such as dopamine (DA) and corticotropinreleasing hormone (CRF), that appear be involved in the regulation of PER2 expression in the BNSTov and CEA $(44,45)$ (see below).

\section{Role of ovarian hormones}

The release of gonadal hormones is influenced by the circadian system (46) and circulating levels of gonadal hormones influence circadian rhythms (47). Because of these interactions and the importance of the limbic forebrain in reproductive physiology and behavior, we examined the role of ovarian hormones in the rhythms of PER2 in the limbic forebrain of female rats. We 
found that the rhythms of PER2 in BNSTov and CEA are strongly affected by the estrous cycle and by estrogen, whereas rhythms in the SCN, BLA and DG are unaffected (48). In males removal of the gonads blunts the expression of PER2 in BNSTov and CEA.

These findings on the role gonadal hormones, taken together with those on glucocorticoids and thyroid hormones indicate that the oscillations of PER2 expression in different regions of the limbic forebrain are differentially sensitive and can be separately modulated by circulating hormones, underscoring the complexity and diversity of mechanisms involved in regulation of PER2 expression in the brain. Furthermore, they provide an important clue to understanding how changes in motivational or emotional state could disrupt tissue specific rhythms within the limbic forebrain and thus the synchrony between rhythms in different regions and, importantly, uncouple them from the rhythm of the SCN.

\section{Modulation of PER2 expression by dopamine and CRF}

We have begun to explore the role of dopamine and corticotropin-releasing factor (CRF) on PER2 expression in the BNSTov and CEA. These two structures receive a dense dopaminergic input (49) and share many anatomical, morphological and chemical features (50-52) including high concentrations of CRF-expressing cells. Dopamine has been implicated in the effect of stress on Fos expression the BNSTov and CEA in rats (53) and has been proposed to play a role in the regulation of CRF mRNA in these regions (54). We found that 
selective unilateral dopamine depletion using microinjections of the neurotoxin, 6hydroxydopamine (6-OHDA) into the medial forebrain bundle reduced the levels of expression of PER2 in the BNSTov and CEA ipsilateral to the lesion, suggesting that dopamine is involved in the regulation of PER2 expression (55).

We have found as well that such lesions of the dopaminergic input to these regions substantially decrease CRF immunoreactivity in both BNSTov and CEA, though not in the paraventricular nucleus (PVN) of the hypothalamus (56). To study the role of CRF in the regulation of PER2 expression, we examined the effect of targeted silencing of CRF gene using long double-stranded RNAmediated RNA interference (57). We found that suppression of CRF expression by microinfusions of dsRNA against CRF into the BNSTov reduces the level of PER2, suggesting that CRF containing cells participate in the regulation of PER2 expression and may indirectly mediate the effects of dopamine as well as of stress and possibly of corticosterone on rhythms of PER2 expression in this structure (58). It is of note that we found previously that CRF and PER2 do not colocalize in these regions, suggesting that involvement of CRF in the expression of PER2 depends on intercellular signally and not the direct action of CRF on transcription of the Per2 gene.

\section{Stress-induced changes in PER2 expression}

Although stress is known to affect circadian behavioral and physiological rhythms $(1,59)$, there is limited evidence concerning the effects of stress, per se, on clock gene expression in the brain. In one study in mice, both acute restraint and 
systemic immune challenge (lipopolysaccharide, LPS) were found to induce the expression of the Per1 gene in the hypothalamic paraventricular nucleus (PVN) (19). Per1 expression in the SCN was not affected and there was no change in the expression of Per2 in either the PVN or SCN, suggesting that acute stressinduced changes in clock genes is both selective and region specific. In another study carried out in neonatal rats, periodic absence of nursing mothers for several days altered the expression of Per1 and Per2 in the SCN (26). We have evidence that exposure to chronic intermittent restraint stress can disrupt the rhythms of PER2 expression in the limbic forebrain, but we have yet to determine which if any of the variables described above is responsible (60).

\section{Synchronization of limbic forebrain PER2 rhythms by restricted feeding.}

Feeding time is a powerful synchronizer of behavioral and physiological circadian rhythms and rhythms in expression of clock genes (61). In nocturnal rodents, feeding that is restricted to the daytime has been shown to uncouple the molecular rhythms of the SCN from the rhythms in the periphery and in some brain regions $(21,22,62)$. Such feeding schedules induce characteristic food anticipatory behavioral and physiological rhythms (e.g., rhythms in behavioral arousal and locomotor activity, body temperature, circulating corticosterone) that are independent of the SCN (63). Importantly, the anatomical location and molecular composition of the oscillators that mediate the effect of restricted feeding on anticipatory rhythms remain elusive. Recent work has focused attention on the dorsomedial hypothalamic nucleus $(\mathrm{DMH})$, which participates in 
the dissemination of circadian rhythmicity from the SCN , and on the clock gene Per2 (64-67).

We are using restricted feeding schedules to study how perturbation of motivational state and energy balance affect the expression of PER2 in the limbic forebrain. In our recent work on scheduled restricted feeding, we found that the rhythmic expression of PER2 in all regions of the limbic forebrain studied is critically sensitive to fluctuations in nutritional state. Specifically, daytime restricted feeding synchronizes the rhythms of PER2 in BNSTov, CEA, BLA and DG and uncouples them from the rhythm of the $\operatorname{SCN}(23,24)$. This finding stands in contrast to the selective effects of hormonal manipulations, which affect only BNSTov and CEA, and points to a critical role for energy balance in the maintenance and integration of circadian rhythms in the brain. Interestingly, we found as well that restricted feeding can reverse the effect of adrenalectomy on PER2 rhythms in BNSTov and CEA and synchronize them with the rhythms in the BLA and DG (68).

Unlike the effect of scheduled restricted feeding, scheduled restricted access to treats such as sucrose, saccharine, or a highly palatable liquid diet, in the absence of food deprivation, has no effect on PER2 expression in the limbic forebrain $(23,24)$ although anticipatory behavior changes can be seen. These findings suggest that changes in PER2 rhythms in the regions studied are triggered by the daily alleviation of a negative metabolic state associated with restricted feeding and are relatively insensitive to incentive properties of the substance or to anticipatory behavioral changes associated with such scheduled 
treats $(23,24)$. Importantly, because restricted feeding and restricted access to a treat induce equally strong patterns of Fos expression in the limbic forebrain, we are able to conclude that the absence of the effect of scheduled treat on PER2 rhythms is not due to failure of the treat-related signals to reach these forebrain regions (23).

\section{Summary and conclusions}

All aspects of behavior and physiology exhibit some degree of circadian rhythmicity under the control of the master circadian clock located in the SCN. In recent years following the discovery of clock genes, it has become evident that many brain regions outside the SCN contain circadian oscillators, which, though subordinate the SCN, can be influenced by behavioral and physiological processes independent of the SCN. Here we reviewed evidence concerning the nature and regulation of such subordinate oscillators in four regions of the limbic forebrain known to play roles in the regulation of motivational and emotional states. Using PER2 clock protein as a marker, we found two rhythmic patterns of expression, one in BNSTov and CEA that is uniquely in phase with the rhythm in the SCN, and the other, as is more common in the brain, in BLA and DG that is opposite in phase with that in the SCN. The uniqueness of the rhythms in the BNSTov and CEA is also expressed in their sensitivity to adrenal, gonadal and thyroid hormones. These rhythms can be altered or even completely blunted by hormonal manipulations that do not in any way affect the PER2 rhythm in the SCN. One manipulation that powerfully affects PER2 rhythms throughout the limbic forebrain is a daily restricted-feeding schedule. Such feeding schedules, 
known to entrain behavioral rhythms, not only entrain the PER2 rhythms in these regions, but furthermore synchronizes their phase. Thus all of these motivational manipulations can uncouple tissue-specific subordinate oscillators from the master circadian clock.

A major task that remains is to identify the functions of PER2 and its rhythms in the limbic forebrain. One idea is that PER2, in its capacity as an essential clock regulator, plays an important role in the maintenance of the functional integrity of these limbic regions. The fact that the rhythms of PER2 in these regions are vulnerable and sensitive to a host of manipulations that do not directly affect the SCN clock, suggests further that they can integrate information from the primary circadian time-keeper in the SCN with information about physiological and motivational state. Finally, it is also possible that PER2 has cellular functions independent of its role in clocks, per se. For example, evidence from Per2 mutant mice indicates that PER2 participates in behavioral processes such as sensitization to the behavioral activating effects of cocaine (69) and alcohol preference (70). However, such mutations eradicate PER2 expression throughout the brain and body, making it impossible to localize the actions of PER2 or to determine whether the effects are due to disruptions of circadian rhythms or simply to the lack of the gene. Whatever the case, uncovering the functions of PER2 and its rhythms within specific brain regions will ultimately depend on being able to disrupt PER2 and its oscillations in a region-specific manner. Recently, we have begun to use RNAi-mediated knockdown of Per2 to address this issue and find that we can disrupt free running locomotor activity 
rhythms in rats using dsRNA to transiently suppress PER2 in the SCN (71).

Work is now in progress that we hope will shed light on functions of PER2 and its rhythms in these motivationally significant regions of the limbic forebrain.

\section{References}

1. Gorka Z, Moryl E, Papp M (1996): Effect of chronic mild stress on circadian rhythms in the locomotor activity in rats. Pharmacol Biochem Behav. 54:229-234.

2. Brodan V, Khun E, Veselkova A, Kaucka J (1982): The effect of stress on circadian rhythms. Czech Med. 5:1-8.

3. Hallonquist JD, Goldberg MA, Brandes JS (1986): Affective disorders and circadian rhythms. Can J Psychiatry. 31:259-272.

4. Amir S, Stewart J (1998): Conditioned fear suppresses light-induced resetting of the circadian clock. Neuroscience. 86:345-351.

5. Funk D, Amir S (1999): Conditioned fear attenuates light-induced suppression of melatonin release in rats. Physiol Behav. 67:623-626.

6. Amir S, Stewart J (1999): The effectiveness of light on the circadian clock is linked to its emotional value. Neuroscience. 88:339-345.

7. Amir S, Stewart J (1999): Conditioned and unconditioned aversive stimuli enhance light-induced fos expression in the primary visual cortex. Neuroscience. 89:323-327.

8. Amir S, Lamont EW, Robinson B, Stewart J (2004): A circadian rhythm in the expression of PERIOD2 protein reveals a novel SCN-controlled oscillator in 
the oval nucleus of the bed nucleus of the stria terminalis. J Neurosci. 24:781790.

9. Lamont EW, Robinson B, Stewart J, Amir S (2005): The central and basolateral nuclei of the amygdala exhibit opposite diurnal rhythms of expression of the clock protein Period2. Proc Natl Acad Sci U S A. 102:4180-4184.

10. Hastings MH, Reddy AB, Maywood ES (2003): A clockwork web: circadian timing in brain and periphery, in health and disease. Nat Rev Neurosci. 4:649661.

11. Dardente H, Cermakian N (2007): Molecular circadian rhythms in central and peripheral clocks in mammals. Chronobiol Int. 24:195-213.

12. Sakamoto K, Nagase T, Fukui H, Horikawa K, Okada T, Tanaka H, et al. (1998): Multitissue circadian expression of rat period homolog (rPer2) mRNA is governed by the mammalian circadian clock, the suprachiasmatic nucleus in the brain. J Biol Chem. 273:27039-27042.

13. Shieh KR (2003): Distribution of the rhythm-related genes rPERIOD1, rPERIOD2, and rCLOCK, in the rat brain. Neuroscience. 118:831-843.

14. Abe M, Herzog ED, Yamazaki S, Straume M, Tei H, Sakaki Y, et al. (2002): Circadian rhythms in isolated brain regions. J Neurosci. 22:350-356. 15. Holzberg D, Albrecht U (2003): The circadian clock: a manager of biochemical processes within the organism. J Neuroendocrinol. 15:339-343. 16. Kalsbeek A, Buijs RM (2002): Output pathways of the mammalian suprachiasmatic nucleus: coding circadian time by transmitter selection and specific targeting. Cell Tissue Res. 309:109-118. 
17. Balsalobre A, Marcacci L, Schibler U (2000): Multiple signaling pathways elicit circadian gene expression in cultured Rat-1 fibroblasts. Curr Biol. 10:12911294.

18. Hirota T, Okano T, Kokame K, Shirotani-Ikejima H, Miyata T, Fukada Y (2002): Glucose down-regulates Per1 and Per2 mRNA levels and induces circadian gene expression in cultured Rat-1 fibroblasts. J Biol Chem. 277:4424444251.

19. Takahashi S, Yokota S, Hara R, Kobayashi T, Akiyama M, Moriya T, et al. (2001): Physical and inflammatory stressors elevate circadian clock gene mPer1 mRNA levels in the paraventricular nucleus of the mouse. Endocrinology. $142: 4910-4917$.

20. Yuferov V, Kroslak T, Laforge KS, Zhou Y, Ho A, Kreek MJ (2003):

Differential gene expression in the rat caudate putamen after "binge" cocaine administration: advantage of triplicate microarray analysis. Synapse. 48:157-169. 21. Wakamatsu H, Yoshinobu Y, Aida R, Moriya T, Akiyama M, Shibata S (2001): Restricted-feeding-induced anticipatory activity rhythm is associated with a phase-shift of the expression of mPer1 and mPer2 mRNA in the cerebral cortex and hippocampus but not in the suprachiasmatic nucleus of mice. Eur $J$ Neurosci. 13:1190-1196.

22. Damiola F, Le Minh N, Preitner N, Kornmann B, Fleury-Olela F, Schibler U (2000): Restricted feeding uncouples circadian oscillators in peripheral tissues from the central pacemaker in the suprachiasmatic nucleus. Genes Dev. 14:2950-2961. 
23. Verwey M, Khoja Z, Stewart J, Amir S (2007): Differential regulation of the expression of Period2 protein in the limbic forebrain and dorsomedial hypothalamus by daily limited access to highly palatable food in food-deprived and free-fed rats. Neuroscience. 147:277-285.

24. Waddington Lamont E, Harbour VL, Barry-Shaw J, Renteria Diaz L, Robinson B, Stewart J, et al. (2007): Restricted access to food, but not sucrose, saccharine, or salt, synchronizes the expression of Period2 protein in the limbic forebrain. Neuroscience. 144:402-411.

25. Zambon AC, McDearmon EL, Salomonis N, Vranizan KM, Johansen KL, Adey D, et al. (2003): Time- and exercise-dependent gene regulation in human skeletal muscle. Genome Biol. 4:R61.

26. Ohta H, Honma S, Abe H, Honma K (2003): Periodic absence of nursing mothers phase-shifts circadian rhythms of clock genes in the suprachiasmatic nucleus of rat pups. Eur J Neurosci. 17:1628-1634.

27. Bae K, Jin X, Maywood ES, Hastings MH, Reppert SM, Weaver DR (2001): Differential functions of mPer1, mPer2, and mPer3 in the SCN circadian clock. Neuron. 30:525-536.

28. Davis M, Walker DL, Lee Y (1997): Amygdala and bed nucleus of the stria terminalis: differential roles in fear and anxiety measured with the acoustic startle reflex. Philos Trans $R$ Soc Lond B Biol Sci. 352:1675-1687.

29. Erb S, Salmaso N, Rodaros D, Stewart J (2001): A role for the CRFcontaining pathway from central nucleus of the amygdala to bed nucleus of the stria terminalis in the stress-induced reinstatement of cocaine seeking in rats. 
Psychopharmacology (Berl). 158:360-365.

30. Loewy AD (1991): Forebrain nuclei involved in autonomic control. Prog Brain Res. 87:253-268.

31. Stefanova N, Ovtscharoff W (2000): Sexual dimorphism of the bed nucleus of the stria terminalis and the amygdala. Adv Anat Embryol Cell Biol. 158:III-X, 1-78.

32. Szafarczyk A, Ixart G, Alonso G, Malaval F, Nouguier-Soule J, Assenmacher I (1983): CNS control of the circadian adrenocortical rhythm. $J$ Steroid Biochem. 19:1009-1015.

33. Balsalobre A, Brown SA, Marcacci L, Tronche F, Kellendonk C, Reichardt HM, et al. (2000): Resetting of circadian time in peripheral tissues by glucocorticoid signaling. Science. 289:2344-2347.

34. Makino S, Gold PW, Schulkin J (1994): Effects of corticosterone on CRH mRNA and content in the bed nucleus of the stria terminalis; comparison with the effects in the central nucleus of the amygdala and the paraventricular nucleus of the hypothalamus. Brain Res. 657:141-149.

35. Pompei P, Riftina F, McEwen BS (1995): Effect of adrenal steroids on preproneurokinin-A gene expression in discrete regions of the rat brain. Brain Res Mol Brain Res. 33:209-216.

36. Watts AG, Sanchez-Watts G (1995): Region-specific regulation of neuropeptide mRNAs in rat limbic forebrain neurones by aldosterone and corticosterone. J Physiol. 484 ( Pt 3):721-736.

37. Segall LA, Perrin JS, Walker CD, Stewart J, Amir S (2006): Glucocorticoid 
rhythms control the rhythm of expression of the clock protein, Period2, in oval nucleus of the bed nucleus of the stria terminalis and central nucleus of the amygdala in rats. Neuroscience. 140:753-757.

38. Tronche F, Kellendonk C, Kretz O, Gass P, Anlag K, Orban PC, et al. (1999): Disruption of the glucocorticoid receptor gene in the nervous system results in reduced anxiety. Nat Genet. 23:99-103.

39. Segall LA, Hsu Z, Robinson B, Milet A, Tronche F, Amir S (2006):

Corticosterone acts centrally to maintain the rhythmic expression of the clock protein, PER2, in the oval nucleus of the bed nucleus of the stria terminalis and central nucleus of the amygdala. Neuroscience Meeting Planner Atlanta, GA: Society for Neuroscience. Program No. 459.5.

40. Beasley LJ, Nelson RJ (1982): Thyroid gland influences the period of hamster circadian oscillations. Experientia. 38:870-871.

41. Murakami N, Hayafuji C, Takahashi K (1984): Thyroid hormone maintains normal circadian rhythm of blood corticosterone levels in the rat by restoring the release and synthesis of ACTH after thyroidectomy. Acta Endocrinol (Copenh). 107:519-524.

42. Preitner N, Damiola F, Lopez-Molina L, Zakany J, Duboule D, Albrecht U, et al. (2002): The orphan nuclear receptor REV-ERBalpha controls circadian transcription within the positive limb of the mammalian circadian oscillator. Cell. 110:251-260.

43. Amir S, Robinson B (2006): Thyroidectomy alters the daily pattern of expression of the clock protein, PER2, in the oval nucleus of the bed nucleus of 
the stria terminalis and central nucleus of the amygdala in rats. Neurosci Lett. 407:254-257.

44. Yilmazer-Hanke DM, Hantsch M, Hanke J, Schulz C, Faber-Zuschratter H, Schwegler H (2004): Neonatal thyroxine treatment: changes in the number of corticotropin-releasing-factor (CRF) and neuropeptide Y (NPY) containing neurons and density of tyrosine hydroxylase positive fibers $(\mathrm{TH})$ in the amygdala correlate with anxiety-related behavior of wistar rats. Neuroscience. 124:283-297. 45. Peterson AL, Gilman TL, Banks ML, Sprague JE (2006): Hypothyroidism alters striatal dopamine release mediated by $3,4-$ methylenedioxymethamphetamine (MDMA, ecstasy). Synapse. 59:317-319. 46. Wiegand SJ, Terasawa E (1982): Discrete lesions reveal functional heterogeneity of suprachiasmatic structures in regulation of gonadotropin secretion in the female rat. Neuroendocrinology. 34:395-404.

47. Albers HE (1981): Gonadal hormones organize and modulate the circadian system of the rat. Am J Physiol. 241:R62-66.

48. Perrin JS, Segall LA, Harbour VL, Woodside B, Amir S (2006): The expression of the clock protein PER2 in the limbic forebrain is modulated by the estrous cycle. Proc Natl Acad Sci U S A. 103:5591-5596.

49. Hasue RH, Shammah-Lagnado SJ (2002): Origin of the dopaminergic innervation of the central extended amygdala and accumbens shell: a combined retrograde tracing and immunohistochemical study in the rat. $J$ Comp Neurol. 454:15-33.

50. De Souza EB, Insel TR, Perrin MH, Rivier J, Vale WW, Kuhar MJ (1985): 
Corticotropin-releasing factor receptors are widely distributed within the rat central nervous system: an autoradiographic study. J Neurosci. 5:3189-3203. 51. Fallon JH, Leslie FM (1986): Distribution of dynorphin and enkephalin peptides in the rat brain. $J$ Comp Neurol. 249:293-336.

52. Ju G, Swanson LW, Simerly RB (1989): Studies on the cellular architecture of the bed nuclei of the stria terminalis in the rat: II. Chemoarchitecture. J Comp Neurol. 280:603-621.

53. Kozicz T (2002): Met-enkephalin immunoreactive neurons recruited by acute stress are innervated by axon terminals immunopositive for tyrosine hydroxylase and dopamine-alpha-hydroxylase in the anterolateral division of bed nuclei of the stria terminalis in the rat. Eur J Neurosci. 16:823-835.

54. Day HE, Vittoz NM, Oates MM, Badiani A, Watson SJ, Jr., Robinson TE, et al. (2002): A 6-hydroxydopamine lesion of the mesostriatal dopamine system decreases the expression of corticotropin releasing hormone and neurotensin mRNAs in the amygdala and bed nucleus of the stria terminalis. Brain Res. 945:151-159.

55. Verwey M, Oudhini A, Waddington Lamont E, Robinson B, Amir S (2006): Dopamine modulates Period2 expression in the oval nucleus of the bed nucleus of the stria terminalis and the central nucleus of the amygdala. Neuroscience Meeting Planner Atlanta, GA: Society for Neuroscience. Program No. 459.6. 56. Stewart J, Horton C, Robinson B, Amir S (2008): Unilateral loss of dopaminergic innervation suppresses the expression of CRF-IR in the ipsilateral oval nucleus of the bed nucleus of the stria terminalis and the central nucleus of 
the amygdala in the rat. Neuroscience Meeting Planner Washington, DC: Society for Neuroscience Program No. 767.21.

57. Bhargava A, Dallman MF, Pearce D, Choi S (2004): Long double-stranded RNA-mediated RNA interference as a tool to achieve site-specific silencing of hypothalamic neuropeptides. Brain Res Brain Res Protoc. 13:115-125.

58. Bhargava A, Idumalla PS, Robinson B, Stewart J, Amir S (2006): Long double-stranded RNA-mediated targeted silencing of corticotropin-releasing hormone diminishes the expression of the clock protein PER2 in the oval nucleus of the bed nucleus of the stria terminalis in rats. Neuroscience Meeting Planner Atlanta, GA: Society for Neuroscience. Program No. 459.4.

59. Meerlo P, Sgoifo A, Turek FW (2002): The effects of social defeat and other stressors on the expression of circadian rhythms. Stress. 5:15-22.

60. Robinson B, Harbour VL, Amir S (2005): Repeated immobilization stress disrupts circadian rhythms of PER2 expression in the limbic forebrain. Neuroscience Meeting Planner Washington, DC: Society for Neuroscience Program No. 60.7 .

61. Mendoza J (2007): Circadian clocks: setting time by food. $J$ Neuroendocrinol. 19:127-137.

62. Challet E, Caldelas I, Graff C, Pevet P (2003): Synchronization of the molecular clockwork by light- and food-related cues in mammals. Biol Chem. 384:711-719.

63. Mistlberger RE (1994): Circadian food-anticipatory activity: formal models and physiological mechanisms. Neurosci Biobehav Rev. 18:171-195. 
64. Mieda M, Williams SC, Richardson JA, Tanaka K, Yanagisawa M (2006): The dorsomedial hypothalamic nucleus as a putative food-entrainable circadian pacemaker. Proc Natl Acad Sci U S A. 103:12150-12155.

65. Gooley JJ, Schomer A, Saper CB (2006): The dorsomedial hypothalamic nucleus is critical for the expression of food-entrainable circadian rhythms. Nat Neurosci. 9:398-407.

66. Landry GJ, Simon MM, Webb IC, Mistlberger RE (2006): Persistence of a behavioral food-anticipatory circadian rhythm following dorsomedial hypothalamic ablation in rats. Am J Physiol Regul Integr Comp Physiol. 290:R1527-1534.

67. Feillet CA, Ripperger JA, Magnone MC, Dulloo A, Albrecht U, Challet E (2006): Lack of food anticipation in Per2 mutant mice. Curr Biol. 16:2016-2022.

68. Segall LA, Verwey M, Amir S (2008): Timed restricted feeding restores the rhythms of expression of the clock protein, Period2, in the oval nucleus of the bed nucleus of the stria terminalis and central nucleus of the amygdala in adrenalectomized rats. Neuroscience. 157:52-56.

69. Abarca C, Albrecht U, Spanagel R (2002): Cocaine sensitization and reward are under the influence of circadian genes and rhythm. Proc Natl Acad Sci U S A. 99:9026-9030.

70. Spanagel R, Pendyala G, Abarca C, Zghoul T, Sanchis-Segura C, Magnone MC, et al. (2005): The clock gene Per2 influences the glutamatergic system and modulates alcohol consumption. Nat Med. 11:35-42.

71. Gavrila AM, Robinson B, Hoy J, Stewart J, Bhargava A, Amir S (2008): Double-stranded RNA-mediated suppression of Period2 expression in the 
suprachiasmatic nucleus disrupts circadian locomotor activity in rats. Neuroscience. 154:409-414. 


\section{$\underline{\text { Acknowledgement }}$}

Supported by Fonds de la recherche en santé Québec (FRSQ), Canadian Institutes of Health Research (CIHR), the Natural Sciences and Engineering Research Council of Canada (NSERC), the Concordia University Research Chairs Program (CRUC).

\section{$\underline{\text { Financial Disclosures }}$}

The authors report no biomedical financial interests or potential conflict of interest. 


\section{Figure caption}

\section{Figure 1.}

Top: Rhythms of PER2 expression in the suprachiasmatic nucleus (SCN), oval nucleus of the bed nucleus of the stria terminalis (OV), central nucleus of the amygdala (CEA), basolateral nucleus of the amygdala (BLA) and dentate gyrus (DG). Symbols indicate means of PER2-IR cells in groups of rats killed at different Zeitgeber times ( $n=4-6$ per group). Figure redrawn from published data $(8,9)$. Bottom: Photomicrographs showing examples of PER2 expression in the SCN, BNSTov, CEA, BLA and DG in rats killed at ZT1 or ZT13. 
Figure 1

Click here to download high resolution image
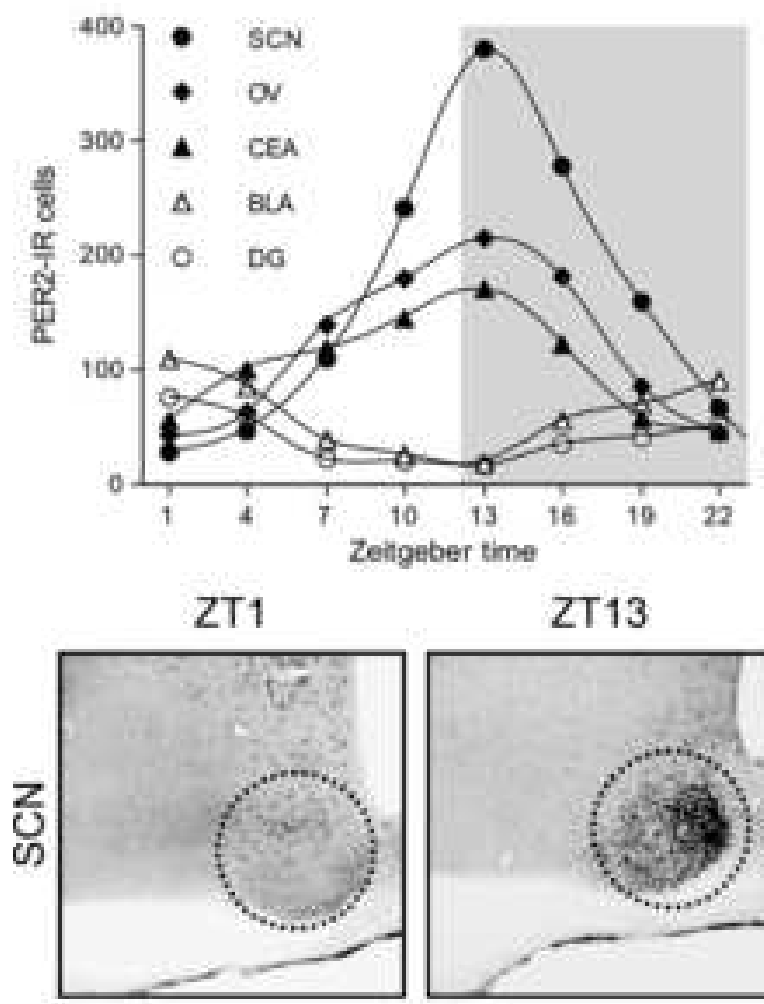

ZT13
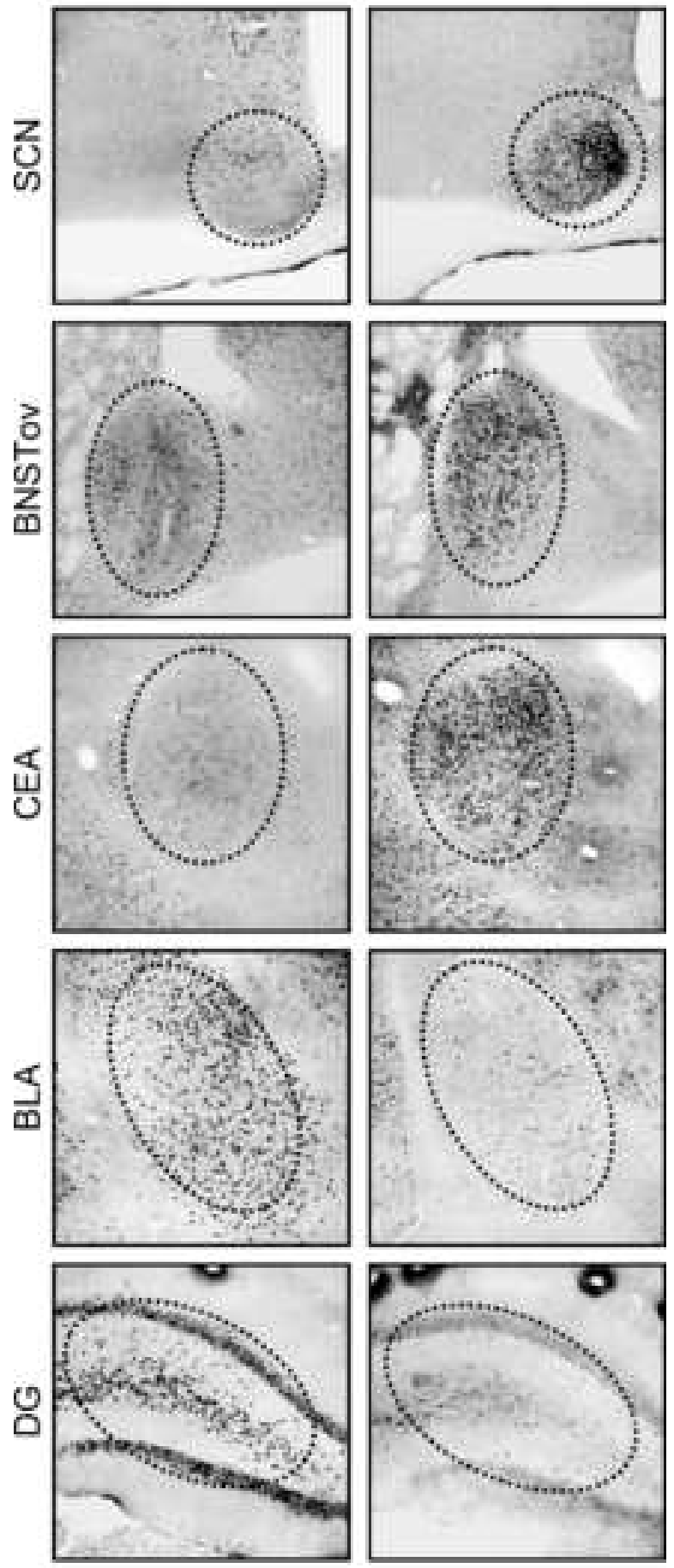